\title{
Heterogeneity of deprivation within very small areas
}

\author{
C Salmond, P Crampton
}

J Epidemiol Community Health 2002;56:669-670

$A^{\prime}$ rea based measures of deprivation are used for resource allocation and research. In both these uses there is continuing debate about the likely proportion of deprived people who would be missed if area based indices were used for interventions or targeting resources. ${ }^{1-4}$ This report uses the same source deprivation variables at area and individual levels to quantify the degree of deprivation heterogeneity within very small areas using data from the New Zealand 1996 census.

\section{METHODS AND RESULTS}

Area deprivation is characterised by the NZDep96 index of deprivation for small areas. The small areas were constructed from one, or several neighbouring, "meshblocks" and 91.5\% contained between 100 and 200 people. The small areas were designed to minimise local heterogeneity while yielding a robust measure of deprivation in the neighbourhood.

NZDep96 is derived from age/sex standardised proportions of people in the small area with each of nine characteristics related to deprivation: having no access to a telephone; aged 18-59 receiving a means tested benefit; aged 18-59 unemployed; living in households with equivalised income (that is, adjusted to control for household composition) below an income threshold; having no access to a car; aged $<60$ living in a single parent family; aged 18-59 without any qualifications; not living in family owned home; living in households below an equivalised bedroom occupancy threshold. ${ }^{5}$ Proportions were calculated from unrounded census data (see Acknowledgement). The proportions were age/sex standardised because age and sex may be risk factors for, but are not direct markers of, deprivation; and because age and sex are usually included separately as components of resource allocation formulas or as potential confounders in epidemiological analyses. The NZDep96 index is the first principal component of the nine adjusted proportions (essentially, the weighted sum of these nine variables that has the largest variance of any such sum) thus maximally ordering the areas along a deprivation continuum. The distribution of NZDep96 scores was also split into deciles, with each decile representing exactly one tenth of the small areas, and close to one tenth of the population.

An index of deprivation for 1.52 million "working age" people (18-59 years) with complete data was constructed similarly to the NZDep96 index but using binary deprivation indicators, scored 1 if the deprivation characteristic was present, and 0 otherwise. An eight point ordinal scale was defined to have value 0 for the first principal component score indicating people with no deprivation characteristic (32.8\%); value 1 for the next set of scores up to, but not including, the minimum score of anyone with two or more deprivation characteristics $(28.9 \%$ had just one characteristic, and may not be deprived in any meaningful way); and values $2,3, . ., 7$ for the sixths of the remaining distribution. A separate index was created for each sex in two age groups of similar size (18-39 and 40-59 years). Combined into an index for people 18-59 years, the index thus describes the socioeconomic deprivation of people relative to others of the same sex at a broadly similar stage in their life cycle.

Given that about $60 \%$ of the population have little or no deprivation as described by the nine deprivation variables, it is clear that they cannot all live in places characterised as being in the one or two least deprived deciles of area deprivation ( fig 1). Even within the most deprived $10 \%$ of small areas, one in ten working aged people (10.8\%) have none of the measured personal characteristics of deprivation (top row of circles). Conversely, $4.1 \%$ of working aged people in the least deprived $10 \%$ of small areas are classified personally as being among the $6.5 \%$ most multiply deprived in the country (bottom row).

For maximum effectiveness, targeting of health resources and interventions requires a mix of area based and individual approaches. For example, (fig 1) if the three most deprived deciles of NZDep96 were used for targeting, comprising one quarter $(14.0 \%+11.3 \%)$ of the study population, these areas

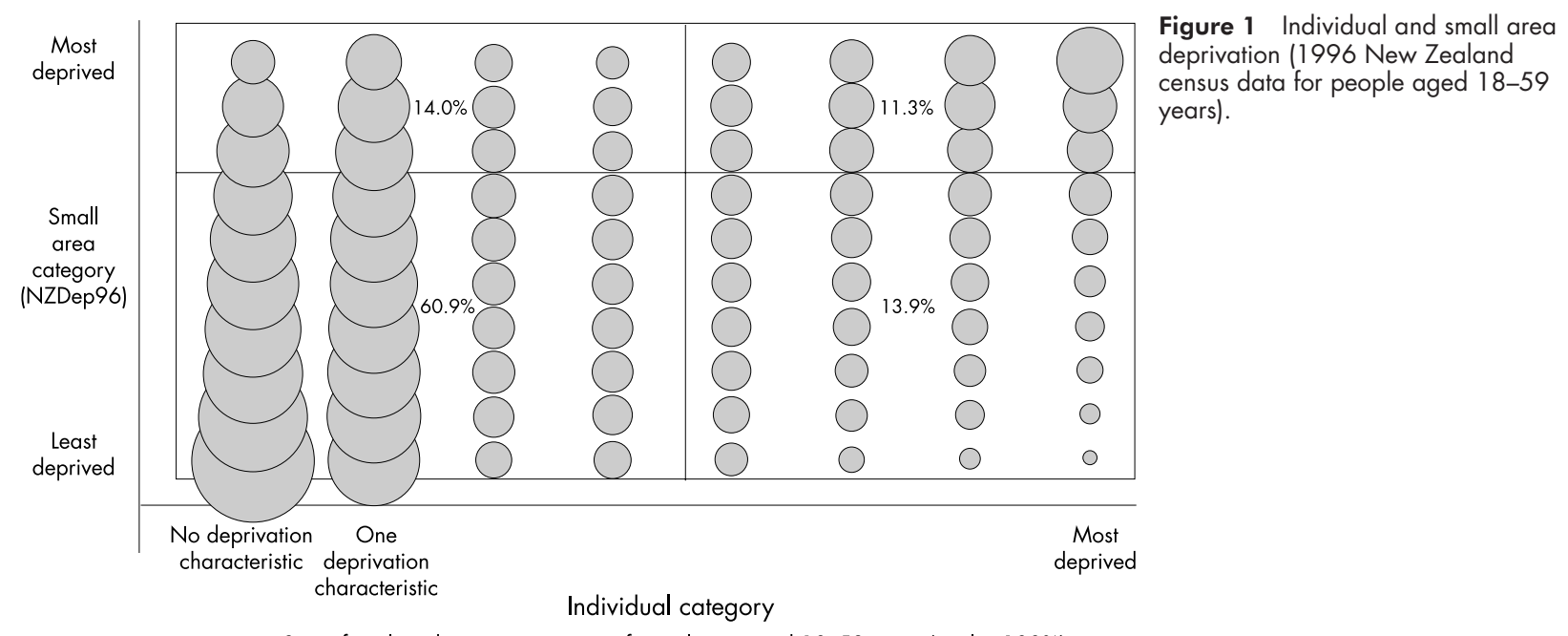

Size of circle indicates percentage of population aged $18-59$ years (total $=100 \%$ ) 
would miss $13.9 \%$ - over half of the quarter of the study population who are individually the most multiply deprived $(13.9 \%+11.3 \%)$.

\section{COMMENT}

We have used the same variables at individual and area levels to highlight and affirm the argument that the selective targeting of resources on an area basis-even very small areas of around 100-200 people-would miss large proportions of multiply deprived people.

\section{ACKNOWLEDGEMENT}

Access was granted to unrounded census data under a special contract with Statistics New Zealand. The access was granted in a strictly protected environment on Statistics New Zealand premises under supervision of Statistics New Zealand staff. The researcher was bound to preserve the confidentiality of individual respondent data by the same provisions of the Statistics Act 1975 that bind staff of Statistics New Zealand.
Authors' affiliations

C Salmond, P Crampton, Department of Public Health, Wellington School of Medicine and Health Sciences, Wellington, New Zealand

Funding: this research was funded by a grant from the Health Research Council of New Zealand.

Conflicts of interest: none.

Correspondence to: Ms C Salmond, Department of Public Health Wellington School of Medicine and Health Sciences, PO Box 7343, Wellington South, New Zealand; salmond@wnmeds.ac.nz

Accepted for publication 19 March 2002

\section{REFERENCES}

1 McLoone P. Targeting deprived areas within small areas in Scotland: population study. BM 2001;323:374-5.

2 Reijneveld S A. Verheii R A, de Bakker D H. The impact of area deprivation on differences in health: does the choice of the geographical classification matter? J Epidemiol Community Health 2000;54:306-13.

3 Danesh J, Gault S, Semmence, J, et al. Postcodes as useful markers of social class: population based study of 26000 British households. BM 1999;318:843-34

4 Ben-Shlomo Y, Davey Smith G. Commentary: Socioeconomic position should be measured accurately. BM 1999;318:844-5.

5 Salmond C, Crampton P. Deprivation and health. In: Howden-Chapman P. Tobias M, es. Social inequalities in health: New Zealand 1999. Wellington: Ministry of Health, 2000:9-63. (also available at http:/ (www.moh.govt.nz) 